\title{
SOX11 expression as a MRD molecular marker for MCL in comparison with $t(11 ; 14)$ and IGH rearrangement
}

\author{
Małgorzata Szostakowska ${ }^{1,2} \cdot$ Michał Szymczyk $^{3} \cdot$ Kalina Badowska $^{1,2} \cdot$ Barbara Tudek $^{1,4} \cdot$ Anna Fabisiewicz $^{2} \odot$
}

Received: 1 February 2018 / Accepted: 28 February 2018 / Published online: 8 March 2018

(c) The Author(s) 2018. This article is an open access publication

\begin{abstract}
The main cause of death in mantle cell lymphoma (MCL) patients is relapse due to undetermined minimal residual disease (MRD) and therefore monitoring MRD is crucial for making the best treatment decisions. The gold standard method for MRD analysis is the quantitative polymerase chain reaction. The most commonly used molecular markers for measuring MRD in MCL are: $\mathrm{t}(11 ; 14)(\mathrm{q} 13 ; \mathrm{p} 32)$ translocation or CCND1 expression and IGH rearrangement. Such markers can, however, be found in other B cell non-Hodgkin lymphomas. Recent studies demonstrate that SOX11 expression is highly specific for MCL and could be used as a marker for measuring MRD. Moreover, evidence shows that SOXI1 level could be predictive for overall survival (OS) and progression-free survival (PFS). We have measured MRD level in follow-up samples from 27 patients diagnosed with MCL using the molecular markers: $\mathrm{t}(11 ; 14)$, IGH rearrangement and SOX11 expression. We compared all markers by their sensitivity, utility and quantitative range. We also examined the predictive value of SOX11 expression for OS and PFS. SOX11 expression was found to have better specificity, quantitative range and utility than the $\mathrm{t}(11 ; 14)$. The predictive value of SOX11 expression was confirmed. At diagnosis, patients with high SOX11 expression had shorter PFS than patients with low SOX11 expression $\left(p=0.04^{*}\right)$; differences between OS being statistically insignificant. To our best knowledge this is a first study comparing SOX11 with $\mathrm{t}(11 ; 14)$ and IGH rearrangement as markers of MRD level. Moreover, in this study we confirmed that SOX11 is useful in cases when other molecular markers cannot be used.
\end{abstract}

Keywords Minimal residual disease $\cdot$ Mantle cell lymphoma $\cdot$ SOX11 $\cdot$ Molecular marker

\section{Introduction}

Mantle cell lymphoma (MCL) is a rare and incurable disease (5-8\% of all NHL), mostly appearing in males (3:1 males: females) at a median age of 68 years [1]. MCL is characterized by an aggressive course and multiple relapsing.

Anna Fabisiewicz

fabisiewicz@coi.waw.pl

1 Faculty of Biology, University of Warsaw, Miecznikowa 1, 02-096 Warsaw, Poland

2 Department of Molecular and Translational Oncology, Maria Skłodowska-Curie Institute - Oncology Center, Roentgena 5, 02-781 Warsaw, Poland

3 Department of Lymphoproliferative Diseases, Maria Skłodowska-Curie Institute - Oncology Center, Roentgena 5, 02-781 Warsaw, Poland

4 Institute of Biochemistry and Biophysics, Polish Academy of Sciences, Pawińskiego 5A, 02-106 Warsaw, Poland
According to the new World Health Organization (WHO) classification, there are two separate types of MCL:

- Classic MCL with IGH-unmutated B cells and SOX11 expression, which usually follows an aggressive course and can transform to the very aggressive blastoid MCL with activation of certain molecular factors [2].

- Leukemic non-nodal MCL with IGH-mutated B cells, without SOX11 expression, usually having an indolent outcome, but can transform to nodal aggressive MCL with activation of certain molecular factors (e.g., TP53) [2].

Relapse due to undetermined minimal residual disease (MRD) is the main cause of death in MCL patients, thereby making monitoring of MRD crucial. Recent studies have shown that consistent monitoring of MRD can improve treatment decisions [3-7]. MRD level can be also predictive for progression-free survival (PFS) [4-6]. In the MCL Younger trial, MRD positivity before autologous stem cell 
transplantation (ASCT) was highly correlated with shorter PFS [3]. Moreover, in patients being in clinical remission, MRD status estimated in peripheral blood (PB) was prognostic for overall survival (OS) [7]. Therefore, consistent monitoring of MRD in MCL could allow improvements to current treatments and predict clinical relapse, PFS and OS.

The gold standard method for measuring minimal residual disease is the quantitative polymerase chain reaction (qPCR). The most widely used molecular markers for measuring MRD in MCL are: $\mathrm{t}(11 ; 14)(\mathrm{q} 13 ; \mathrm{p} 32)$ translocation or CCND1 expression [8] and IGH rearrangement [9].

The $\mathrm{t}(11 ; 14)(\mathrm{q} 13 ; \mathrm{p} 32)$ translocation which causes cyclin D1 (CCNDI) overexpression can be detected in 90\% MCL cases. CCND1 is one of the proteins that regulates cell cycle thus its upregulation drives B cells to proliferation [10]. It is applied either to diagnostics or to MRD monitoring for MCL [8]. A lack of this overexpression is characterized as a CCND1 negative subtype of MCL [11] and appears in $10 \%$ of MCL cases. Moreover, cyclin D1 overexpression is also found in CLL [12], which has a similar clinical image to leukemic non-nodal MCL. The $\mathrm{t}(11 ; 14)$ translocation is confirmed by fluorescence in situ hybridization (FISH) in 90\% patients, but it is detectable by PCR methods in only $25-40 \%$ of cases [13].

IGH rearrangement is highly specific to each patient. It is used as molecular marker in many B cell neoplasms and allows detection of tumor cells in 80-90\% of patients [14]. However, measuring MRD by IGH rearrangement is laborintensive. It is based on the qPCR assay which demands designing allele-specific oligonucleotide (ASO) PCR primers. Furthermore, it is impossible to distinguish two subtypes of $\mathrm{B}$ cell neoplasms by this method in rare cases of composite lymphoma (CL).

Considering these aforementioned limitations, there is a need to find a specific and sensitive molecular marker for monitoring minimal residual disease in MCL. Recent studies have shown that SOX11 expression is highly specific for MCL [15] and independent of the presence of $t(11 ; 14)$ [16] and thus it can be used as a molecular marker even for the CCND1(-) negative MCL subtype [11]. SOX11 expression is also more specific for MCL than $C C N D 1$ overexpression; being, respectively, found in $90-95 \%$ and $90 \%$ of MCL cases [17]. SOX11 expression also distinguishes two types of MCL: classic from leukemic non-nodal [2] and can be predictive for OS [18, 19]. Evidence has shown that SOX11 expression can be used as a measurement of MRD in MCL [20] with at least the same sensitivity but better specificity than $\mathrm{t}(11 ; 14)[21]$. Therefore, SOX11 could potentially appear as a better molecular marker than $\mathrm{t}(11 ; 14)$. In this study, we compared SOX11 expression, t $(11 ; 14)$ and IGH rearrangement as MRD molecular markers to determine if SOX11 can be used with at least the same sensitivity and specificity as other molecular markers.

\section{Materials and methods}

PB and BM from 34 patients treated in the Department of Lymphoproliferative Diseases, Maria Skłodowska-Curie Institute-Oncology Center were collected: 71 samples at diagnosis and 254 samples during treatment. There were at least two samples from every patient at diagnosis and 3-15 samples were collected from 27 patients during treatment. PB samples from healthy donors served as negative controls. White blood cells (WBC) were isolated by density gradient centrifugation. Samples containing $5 \times 10^{6}$ cells were frozen and stored at $-80{ }^{\circ} \mathrm{C}$. The research protocol was reviewed and approved by the Ethics Committee of Maria Skłodowska-Curie Institute-Oncology Center. Informed consent was obtained from all individual participants included in the study before the collection of biological material.

RNA isolation from cell precipitate was performed with the GeneMATRIX Universal Purification Kit (EURx, Poland) according to the manufacturer's protocol. RNA concentration and purity were measured using the NanoDrop spectrophotometer (ThermoFisher) and FlashGel system (Lonza). A $500 \mathrm{ng}$ of RNA was reverse transcribed (RT) using the NG dART Reverse Transcription Kit (EURx) according to the manufacturer's protocol. cDNA quality was estimated by PCR with GAPDH primers: F: 5'-GAAGGTGAAGGTCGGAGTC-3'; R: 5'-GAA GATGGTGATGGGATTTC-3', using the NG dART RTPCR kit (EURx) according to the manufacturer's protocol. cDNA was diluted to a final volume of $35 \mu \mathrm{l}$ when RT efficiency was low, and to a final $50 \mu$ l volume when RT efficiency was high. Diluted cDNA was stored at $-20{ }^{\circ} \mathrm{C}$ or $+4{ }^{\circ} \mathrm{C}$ if used within $24 \mathrm{~h}$. qPCR was performed according to a highly sensitive and specific qPCR assay for SOX11 measurement previously described by Hamborg et al. (2012) [20], using the SensilFAST Probe Lo-ROX Mix (BioLine) without the uracil-DNA glycosylase (UNG) enzyme.

DNA isolation was performed using the Sherlock AX isolation kit (A\&A Biotechnology) according to the manufacturer's protocol. For IGH rearrangement, PCR and $\mathrm{qPCR}$ reactions were performed according to the BIOMED-2 Concerted Action BMH4-CT98-3936 protocol [9] using 7500 Fast Real Time PCR System (Applied Biosystems, Foster City, CA).

Standard curves and follow-up analysis were done according to van der Velden et al. (2007) [22]. PCR and qPCR reactions for $\mathrm{t}(11 ; 14)$ were performed according to Pott et al. (2013) [23] For four patients, there was a need to design ASO PCR primers.

Sensitivity of molecular markers was defined by the lowest possible dilution detected by qPCR. The 
quantitative measurement range was set up as the lowest detected dilution which had a $\mathrm{Ct}$ lower than that of the negative controls background by three cycles.

MRD level was calculated to allow comparisons between molecular markers to be made. Results from diagnostic samples were set to a $1(100 \%)$ MRD level while MRD level in follow-up samples was calculated as derived proportions.

Statistical analysis was performed on Microsoft-Excel using correlation, linear regression and log-rank testing. Graphs were constructed via GraphPad Prism software (GraphPad Prism Inc.).

\section{Results}

SOX11 expression was measured as a proportion of SOX11 copies/1000 reference genes (RG) copies. Results obtained for patients were very highly variable; from $2 \times 10^{-2}$ SOX11/1000 RG to 353 SOX11/1000 RG. Four patients were diagnosed with blastoid MCL and all four had SOX11 expression higher than 100 SOX11/1000 RG. Eighteen patients with low SOX11 expression in the diagnostic sample were usually diagnosed with less aggressive disease. Three patients with low SOX11 expression in the diagnostic sample diagnosed with aggressive advanced nodal MCL usually had multiple relapses during treatment. Sixteen patients with less than 1 SOX11/1000 RG were excluded from further analysis of MRD.

MRD analysis was performed for: 18 patients (53\%) by SOX11 expression, 9 patients $(26 \%)$ by $\mathrm{t}(11 ; 14)$ and 21 patients (61\%) using IGH rearrangement. Due to low SOX11 expression and poor sensitivity of the $\mathrm{t}(11 ; 14)$ translocation, in 5 patients (15\%) the only molecular marker that could be used was IGH rearrangement. For two patients with a high polyclonal background and low SOXI1 expression, the only molecular marker applicable was $t(11 ; 14)$. Because of the polyclonal background and poor sensitivity of $t(11 ; 14)$ in one patient $(2 \%)$, the only molecular marker that could be used was SOX11 expression.

Correlation between the MRD level measured by $\mathrm{t}(11 ; 14)$ and SOX11 expression was very high, at 0.98 . Sensitivity for both methods was near $1 \times 10^{-5}$ but for $\mathrm{t}(11 ; 14)$ the mean quantitative measurement range was $1.2 \times 10^{-4}$. The difference between MRD level estimated by both markers was not statistically significant, $p>0.98$. For further analysis, we estimated a linear regression model using MRD level measured by SOX11 expression and $\mathrm{t}(11 ; 14)$ (Fig. 1).

When MRD levels were measured in parallel by SOX11 expression and the IGH rearrangement the correlation was also high at 0.89 . The sensitivity of both molecular markers was near $1 \times 10^{-5}$ and for IGH rearrangement the mean quantitative range was $5 \times 10^{-5}$. As in the previous comparison, the difference between MRD level measured by both

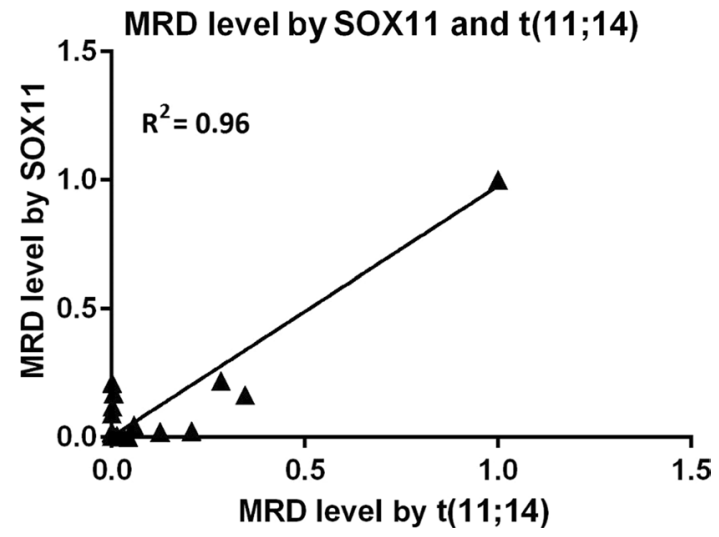

Fig. 1 Linear regression of MRD levels measured by molecular markers of SOX11 expression and $\mathrm{t}(11 ; 14)$ translocation

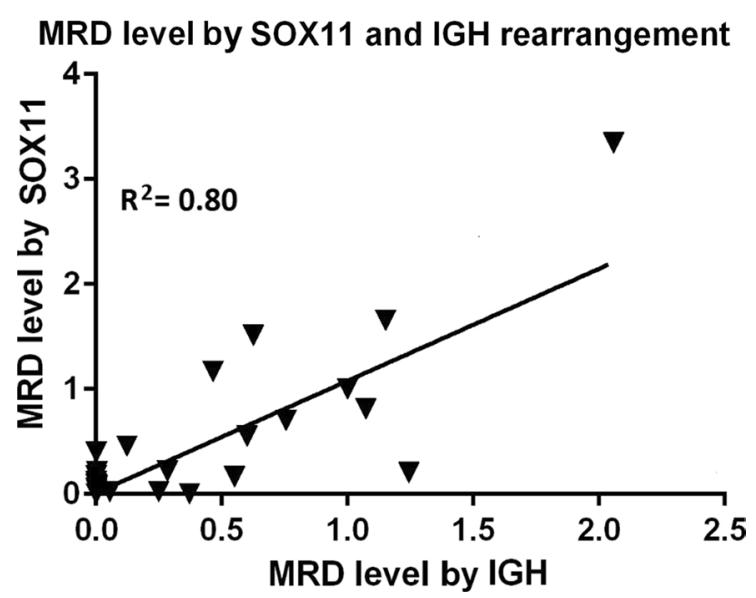

Fig. 2 Linear regression of MRD levels measured by molecular markers of SOX11 expression and IGH rearrangement

markers was not statistically significant, $p>0.79$. Likewise, as before a linear regression model was estimated using MRD level calculated by SOXI1 expression and IGH rearrangement (Fig. 2).

We also compared MRD level obtained from different biological material: PB and BM using the following molecular markers: SOX11 expression and IGH rearrangement. Correlation between PB and BM samples was close at 0.85 for both molecular markers. (Fig. 3).

In samples taken at diagnosis, SOX11 expression was measured in 34 patients. Patients were divided into two groups: SOX $11^{\text {negative/low }}$ with expression $\leq 10$ SOX11 copies/1000 RG copies and SOX1 $1^{\text {intermediate/high }}$ with expression $>10$ SOX11 copies/1000 RG copies. Mean PFS in the $S O X 11^{\text {negative/low }}$ group of patients was $\sim 36$ months but for SOX1 $11^{\text {intermediate/high }}$ it was only $\sim 16$ months (Fig. 4). The difference between mean PFS in the groups is statistically significant $(p=0.04)$. Mean OS in the SOXI $1^{\text {negative/low }}$ group 


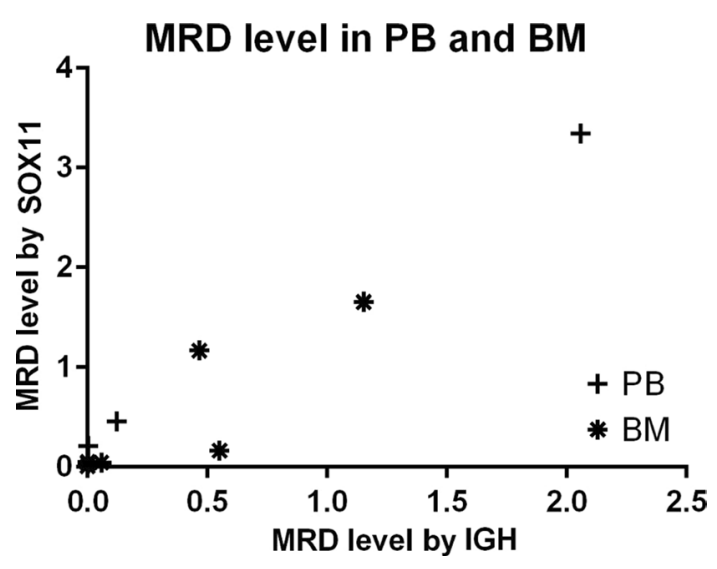

Fig. 3 Comparison of MRD levels for PB and BM samples measured by the following molecular markers: SOX11 expression, IGH rearrangement. BM results are different for both markers with an $87 \%$ similarity in trend

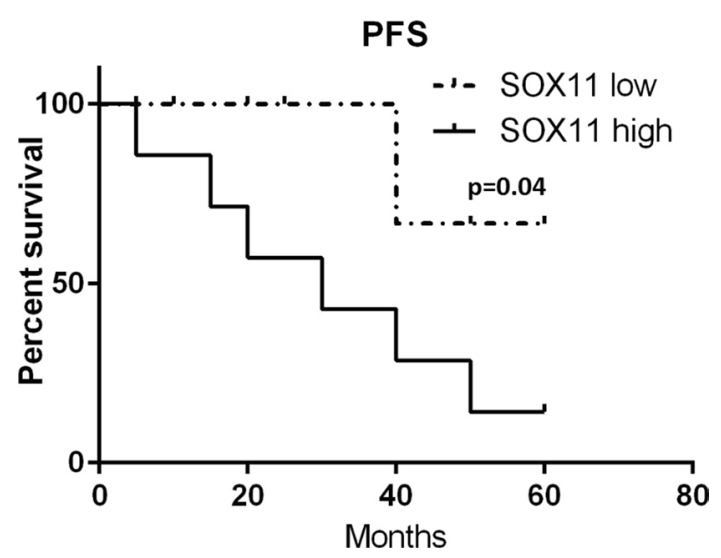

Fig. 4 Progression-free survival in patients from two groups of SOX11 expression: SOX $11^{\text {negative/low }}-S O X 11$ low and SOX$11^{\text {intermediate/high }}-S O X 11$ high. The difference between mean PFS in groups is statistically significant

of patients was $\sim 41$ months, and for the $S O X 11^{\text {intermediate/high }}$ group it was $\sim 30$ months; however, this difference between groups was statistically insignificant $(p=0.09)$ (Fig. 5).

\section{Discussion}

Our study aimed to compare SOX11 expression, $\mathrm{t}(11 ; 14)$ translocation and IGH rearrangement as the qPCR molecular markers for measuring MRD level in MCL. We have demonstrated that all markers have similar sensitivity but the mean quantitative range for $\mathrm{t}(11 ; 14)$ and for IGH rearrangement was lower than that for SOX11 $\left(1.2 \times 10^{-4}, 5 \times 10^{-5}\right.$, $1 \times 10^{-5}$, respectively). For $S O X 11$ expression, the quantitative range was the same as the sensitivity, because there

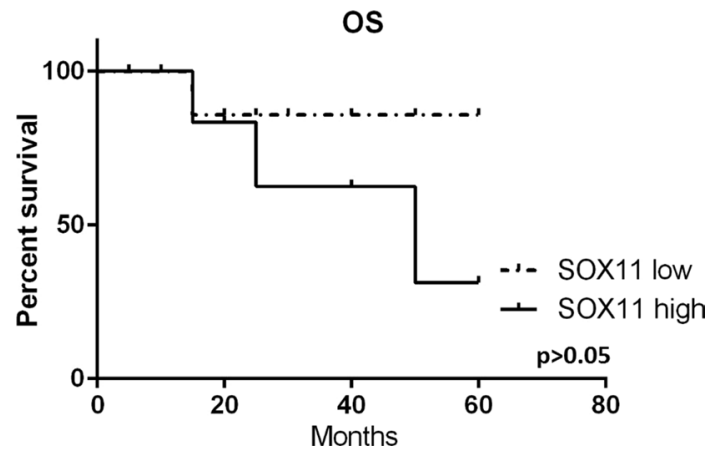

Fig. 5 Overall survival in patients from two groups of SOX11 expression: low and high expression of SOX11. The difference between OS is not statistically significant

was no background from the negative control in any of the reactions. We have therefore concluded that $S O X 11$ expression is a more specific molecular marker than $\mathrm{t}(11 ; 14)$ and IGH rearrangement. Moreover, there were no statistically significant differences between MRD level obtained using molecular markers: $\mathrm{t}(11 ; 14)$ and SOX11 or IGH rearrangement and SOX11.

MRD level measured by SOX11 expression and $\mathrm{t}(11 ; 14)$ translocation were comparable, with correlation of 0.98 . We thus assumed that these molecular markers could be used interchangeably or that even SOX 11 expression can be used as a molecular marker instead of $t(11 ; 14)$. In this study, we applied the commonly used PCR primers designed for the major translocation cluster (MTC) which covers about $40 \%$ of MCL translocations. Insufficient specificity of $\mathrm{t}(11 ; 14)$ primers used in qPCR reactions limits the use of this marker for monitoring MRD to only $25-40 \%$ cases [13]. Our study showed that the $\mathrm{t}(11 ; 14)$ translocation was specific to $26 \%$ patients, while the SOX11 expression to $53 \%$ patients, including one patient with CCND1(-) MCL. Our work confirms previous results suggesting that in comparison with the $\mathrm{t}(11 ; 14)$ translocation, $S O X 11$ expression is a more specific molecular marker with similar sensitivity [20, 21].

Our work showed that MRD level measured by IGH rearrangement and SOX11 expression was comparable, with a correlation coefficient 0.89 . The IGH rearrangement was used as a MRD molecular marker for most patients $(61 \%)$ compared to the other molecular markers: $\mathrm{t}(11 ; 14)$ translocation and SOX11 expression (26 and 53\%, respectively). These results confirm previous assumptions about the utility of the IGH rearrangement [14]. Former work on comparing SOX11 and IGH rearrangement suggests that SOX11 expression could be less sensitive than IGH rearrangement [17]. In our study, SOX11 expression was detectable at very low MRD level in the follow-up samples, especially for patients who had high $S O X 11$ expression at diagnosis $(>10$ SOX11 copies/1000 RG copies). However, for a few patients 
SOX11 expression was not detectable in the follow-up samples at very low MRD level while IGH rearrangement was. All such patients had low SOX11 expression at diagnosis (<10 SOX11 copies/1000 RG copies). Our results confirm previous conclusions, that SOX 11 has a lower sensitivity than IGH rearrangement [17], but only for patients with low SOX11 expression at diagnosis. This may be caused by the fact that during treatment, the expression of SOX11 decreases significantly. In patients with low baseline expression of SOX11, the level of expression during treatment decreases to values undetectable by RT-qPCR. However, in patients in whom the initial expression of SOX11 is high, the decrease in the level of SOXI1 expression during treatment decreases within the limits of detection. Moreover, the difference in the sensitivity of these two markers may be due to the specification of the genetic material. RNA, which is the material for estimation of SOX11 expression, is less stable thus, it degrades faster than DNA. Therefore, when a small amount of material is analyzed, the detectability of DNA is better than RNA.

Our study also investigated the suggested prognostic value $[18,19]$ of $S O X 11$ expression. We compared PFS and OS in groups of patients with low and high SOX11 expression at diagnosis. Patients with high SOX11 expression (>10 SOX11 copies/1000 RG copies) had shorter PFS than patients with low SOX11 expression $(<1$ SOX11/1000 $\mathrm{RG})$. The difference between mean PFS in both groups was $\sim 20$ months and was statistically significant $(p=0.04)$. There was no statistically significant difference between OS for patients with high and low SOX11 expression. Our results confirm the prognostic value of SOX11 expression at diagnosis but only for progression-free survival which contrasts to previous studies in which low SOX11 expression in vitro and in vivo was associated with poor prognosis, shorter OS and PFS $[24,25]$. High expression of SOX11 in tumor cells could lead to a more aggressive disease course $[18,19]$ with increased proliferation and cell survival, by activating FAK and CXCR4 [26]. Therefore, early detection of increasing level of SOX11 expression may be important for improving therapeutic decisions.

Our results confirm high specificity of SOX11 expression as a molecular marker for MRD in MCL. Moreover, the quantitative range of MRD level measured by SOX11 was higher than the quantitative range for that obtained with the $\mathrm{t}(11 ; 14)$ translocation and IGH rearrangement. Furthermore, MRD level obtained with the use of SOX11, $\mathrm{t}(11 ; 14)$ and IGH rearrangement was highly correlated. We therefore hope that SOX11 expression will be used as a molecular marker for measuring MRD in MCL with the same frequency of $t(11 ; 14)$ or IGH rearrangement. However, considering limitations emerging from stability of RNA, for the most efficient estimation of MRD level in MCL patients, SOX11 expression should be used parallelly with
IGH rearrangement. Simultaneous usage of both markers allows to confirm results obtained with SOX11 expression. Moreover, this could permit to estimate MRD level in different subtypes of myelomas in rare cases of composite lymphoma. We also confirmed the prognostic value of SOX11 expression, when high SOX11 expression is correlated with poor prognosis. Considering the discordant results on the prognostic value of SOX11 expression, we think that this subject requires further study, including studies on pathways regulated by SOX11, SOX11 methylation profiles and potential isoforms of SOX11.

Funding This study was supported by the Grant from The Young Haematologists Club of the Polish Society of Haematology and Blood Transfusion (eduGrant 106476).

\section{Compliance with ethical standards}

Conflict of interest The authors declared no conflict of interest.

Ethical approval All procedures performed in studies involving human participants were in accordance with the ethical standards of the institutional and/or national research committee and with the 1964 Helsinki Declaration and its later amendments or comparable ethical standards.

Open Access This article is distributed under the terms of the Creative Commons Attribution 4.0 International License (http://creativeco mmons.org/licenses/by/4.0/), which permits unrestricted use, distribution, and reproduction in any medium, provided you give appropriate credit to the original author(s) and the source, provide a link to the Creative Commons license, and indicate if changes were made.

\section{References}

1. Zhou Y, Wang H, Fang W, et al. Incidence trends of mantle cell lymphoma in the United States between 1992 and 2004. Cancer. 2008;113:791-8.

2. Swerdlow SH, Campo E, Pileri SA, et al. The 2016 revision of the World Health Organization classification of lymphoid neoplasms. Blood. 2016;127:2375-90.

3. Hermine O, Hoster E, Walewski J, et al. Addition of high-dose cytarabine to immunochemotherapy before autologous stem-cell transplantation in patients aged 65 years or younger with mantle cell lymphoma (MCL Younger): a randomised, open-label, phase 3 trial of the European Mantle Cell Lymphoma Network. Lancet. 2016;388:565-75.

4. Kwok M, Rawstron AC, Varghese A, et al. Minimal residual disease is an independent predictor for 10-year survival in CLL. Blood. 2016;128:2770-3.

5. Luskin MR, Stone RM. Can minimal residual disease determination in acute myeloid leukemia be used in clinical practice? J Oncol Pract. 2017;13:471-80.

6. Paietta E. Assessing minimal residual disease (MRD) in leukemia: a changing definition and concept? Bone Marrow Transpl. 2002;29:459-65.

7. Pott CME, Delfau-Larue MH, et al. MRD eradication should be the therapeutic goal in mantle cell lymphoma and may enable 
tailored treatment approaches: results of the intergroup trials of the European MCL Network. Blood. 2014;124:147.

8. Bacher U, Kern W, Haferlach C, et al. Cyclin D1 (CCND1) messenger RNA expression as assessed by real-time PCR contributes to diagnosis and follow-up control in patients with mantle cell lymphoma. Exp Hematol. 2013;41:1028-37.

9. van Dongen JJ, Langerak AW, Bruggemann M, et al. Design and standardization of PCR primers and protocols for detection of clonal immunoglobulin and T-cell receptor gene recombinations in suspect lymphoproliferations: report of the BIOMED-2 concerted action BMH4-CT98-3936. Leukemia. 2003;17:2257-317.

10. Wiestner A, Tehrani M, Chiorazzi M, et al. Point mutations and genomic deletions in CCND1 create stable truncated cyclin D1 mRNAs that are associated with increased proliferation rate and shorter survival. Blood. 2007;109:4599-606.

11. Mozos A, Royo C, Hartmann E, et al. SOX11 expression is highly specific for mantle cell lymphoma and identifies the cyclin D1-negative subtype. Haematologica. 2009;94:1555-62.

12. Abboudi Z, Patel K, Naresh KN. Cyclin D1 expression in typical chronic lymphocytic leukaemia. Eur J Haematol. 2009;83:203-7.

13. Pott C. Minimal residual disease detection in mantle cell lymphoma: technical aspects and clinical relevance. Semin Hematol. 2011;48:172-84.

14. Hoster E, Pott C. Minimal residual disease in mantle cell lymphoma: insights into biology and impact on treatment. Hematology Am Soc Hematol Educ Program. 2016;2016:437-45.

15. Dictor M, Ek S, Sundberg M, et al. Strong lymphoid nuclear expression of SOX11 transcription factor defines lymphoblastic neoplasms, mantle cell lymphoma and Burkitt's lymphoma. Haematologica. 2009;94:1563-8.

16. Chen YH, Gao J, Fan G, Peterson LC. Nuclear expression of sox 11 is highly associated with mantle cell lymphoma but is independent of $\mathrm{t}(11 ; 14)(\mathrm{q} 13 ; \mathrm{q} 32)$ in non-mantle cell B-cell neoplasms. Mod Pathol. 2010;23:105-12.

17. Simonsen AT, Schou M, Sorensen CD, et al. SOX11, CCND1, $\mathrm{BCL} 1 / \mathrm{IgH}$ and IgH-VDJ: a battle of minimal residual disease markers in mantle cell lymphoma? Leuk Lymphoma. 2015;56:2724-7.

18. Fernandez V, Salamero O, Espinet B, et al. Genomic and gene expression profiling defines indolent forms of mantle cell lymphoma. Cancer Res. 2010;70:1408-18.

19. Navarro A, Clot G, Royo C, et al. Molecular subsets of mantle cell lymphoma defined by the IGHV mutational status and SOX11 expression have distinct biologic and clinical features. Cancer Res. 2012;72:5307-16.

20. Hamborg KH, Bentzen HH, Grubach L, et al. A highly sensitive and specific qPCR assay for quantification of the biomarker SOX11 in mantle cell lymphoma. Eur J Haematol. 2012;89:385-94.

21. Simonsen AT, Sorensen CD, Ebbesen LH, et al. SOX11 as a minimal residual disease marker for Mantle cell lymphoma. Leuk Res. 2014;38:918-24.

22. van der Velden VH, Cazzaniga G, Schrauder A, et al. Analysis of minimal residual disease by Ig/TCR gene rearrangements: guidelines for interpretation of real-time quantitative PCR data. Leukemia. 2007;21:604-11.

23. Pott $\mathrm{C}$, Bruggemann M, Ritgen $\mathrm{M}$, et al. MRD detection in B-cell non-Hodgkin lymphomas using Ig gene rearrangements and chromosomal translocations as targets for real-time quantitative PCR. Methods Mol Biol. 2013;971:175-200.

24. Wang X, Asplund AC, Porwit A, et al. The subcellular Sox 11 distribution pattern identifies subsets of mantle cell lymphoma: correlation to overall survival. Br J Haematol. 2008;143:248-52.

25. Yang W, Wang Y, Yu Z, et al. SOX11 regulates the pro-apoptosis signal pathway and predicts a favorable prognosis of mantle cell lymphoma. Int J Hematol. 2017;106:212-20.

26. Balsas P, Palomero J, Eguileor A, et al. SOX11 promotes tumor protective microenvironment interactions through CXCR4 and FAK regulation in mantle cell lymphoma. Blood. 2017;130:501-13. 